\title{
Large Eddy Simulation of Flows Associated with Offshore Oil and Gas Pipeline
}

\author{
Z. Nizamani ${ }^{1, *}$ and A. Nakayama ${ }^{1}$ \\ ${ }^{1}$ Department of Environmental Engineering, Universiti Tunku Abdul Rahman (UTAR), P.C. 31900, \\ Kampar, Perak Darul Ridzuan, Malaysia
}

\begin{abstract}
Fluid structure interaction (FSI) applications are of wide range from offshore fixed and floating structures to offshore pipelines. Reynolds Averaged Navier Stoke (RANS) solution has limitation for unsteady and turbulent flow modelling. A possible approach is Large Eddy Simulation (LES) and it is applied to flows past a circular cylinder located far above, near and on a flat seabed. The Reynolds number considered is based on the real situation off Malaysia Coast and is sub-critical around 105. Hydrodynamic quantities in terms of mean pressure are predicted and vortex shedding mechanism is evaluated. The results are validated by comparing the simulation and experimental previous studies.
\end{abstract}

\section{Introduction}

One of the most significant problems in ocean engineering is flow around a circular cylinder. This flow represents flow around bluff body which carries significant interest in many engineering applications like hydrodynamic loading on marine risers, jacket legs, marine pipeline, FPSO and tension leg platforms moorings [1,2]. The design current velocity being used in offshore Malaysia varies for three different regions of Malaysia for operational and extreme conditions and it lies between 0.3 to $0.81 \mathrm{~m} / \mathrm{sec}$ respectively.

The Reynolds number based on the pipe diameter and the mean current velocity varies from $8.1 \times 10^{4}$ to $2.1 \times 10^{5}$ in this region which indicates that the flow is in high subcritical range or near critical. The experimental work of obtaining detailed flow quantities at such high Reynolds number is very difficult and there are only few cases where it has been reported in literature [3]. This variation of Reynolds number makes it necessary to evaluate the flow around the pipe in the actual range. In this study the flow around and in the near wake of circular pipe is analysed with the expected range of the Reynolds number and other parameters such as the distance to the seabed. The grid system used in the numerical analysis is fixed rectangular mesh in Cartesian coordinates. The results could be enhanced if grid resolution is increased. Boundary geometries are of common shape. We assume that the bottom boundary is not consisted of loose materials like mud sediment. In the next section fundamental equations of motion and numerical models that are used in the analysis

*Corresponding author: zafarullah@utar.edu.my 
are discussed. Then a LES method with a wall model is described. Its accuracy is verified by comparing with two cases of experimental data for the uniform flow. The results show that LES captures correctly the boundary layer separation and mean pressure distribution prediction at high Reynolds number of $10^{5}$.

\section{Previous work}

Flow surrounding a circular cylinder has been studied by researchers for its flow phenomena and engineering applications in designing jacket legs, mooring lines of spar platforms, Floating production system and operations (FPSO), pipelines etc. Recent studies on flow around circular cylinder in uniform flow at high Reynolds number are reported by [4 - 12]. Due to the nature of many issues, there still remain many aspects of the flow that need further investigation [10]. LES is a best method available currently for determining turbulent flow characteristics. There are some advantages using fixed rectangular grids which present flow in natural and complex areas as compared to unstructured grid (boundary) following a mesh system or mesh free method. Conventional Computational Fluid Dynamics (CFD) method of RANS or advanced methods such as DNS or LES are based on basic governing equation of Fluid Dynamics. Incompressible viscous flow of Newtonian fluid is considered in this study.

\subsection{Large Eddy Simulation (LES)}

In this method large eddies are computed (resolved) and smallest sub-gird-scale eddies are model[11]. This is based on Kolmogorov's theory on turbulence which makes the assumption that large eddies of the flow are dependent on the flow geometry, whereas smaller eddies are self-similar and have a universal character. Large eddies are solved explicitly and effect of smaller eddies is modelled on large eddies. In this model large scale motions of the flow are evaluated similar to DNS method and effect of smaller universal scales on sub-grid scale model are modified. In this method it can be assumed that large scale is solved using DNS and small scale as RANS method.

Boundary conditions are very important in RANS and LES methods for all applications whereas initial conditions have secondary importance in these methods. This condition plays significant role in wall bounded flows of higher order quality but it comes with high computational costs. Wall boundary conditions are used in fluid flows of practical applications. The wall changes the fluid dynamics in an important manner this is due to the fact that in the near wall region, the sharp gradient for flow velocity will create large shear stresses as compared to far away regions from wall boundary. This is due to large viscosity stresses in the near wall region. Near wall flow under turbulence loading is still under study because study in this region involves a rapid change of turbulent time and length scales. CFD modelling requires wall boundary conditions to be properly defined for flow.

\subsection{Computational Model}

In this case an offshore oil and gas pipeline is selected for the analysis of LES model. LES model can be significantly The LES model for offshore pipeline has been discussed by some of the authors $[1,12]$. The LES model for circular cylinder general has been performed by $[13,14]$. Spatial and time wise scale of flow are very large and spacing of grid points and time step are made possible to resolve dissipation scales by dividing flow region into cubes. The order of cube is smaller in length for large scale flow and a fraction of 
turbulent fluctuation can be resolved to get mean flow and turbulent stresses. Filtered flow of this scale can represent overall characteristics of simulated flow.

\subsubsection{Governing Equations}

Filtered equations of motion and continuity for spatially filtered velocity components $(\mathrm{u}, \mathrm{v}, \mathrm{w})$ and pressure $\mathrm{p}$ are shown in Equation 1,

$$
\begin{aligned}
\frac{\partial u}{\partial t}+\frac{\partial u^{2}}{\partial x}+\frac{\partial u v}{\partial y}+\frac{\partial u w}{\partial z}= & -\frac{1}{\rho} \frac{\partial p}{\partial x}+\frac{\partial}{\partial x}\left(v \frac{\partial u}{\partial x}+\tau_{x x}\right)+\frac{\partial}{\partial y}\left(v \frac{\partial u}{\partial y}+\tau_{y x}\right) \\
& +\frac{\partial}{\partial z}\left(v \frac{\partial u}{\partial z}+\tau_{z x}\right) \\
\frac{\partial v}{\partial t}+\frac{\partial u v}{\partial x}+\frac{\partial v^{2}}{\partial y}+\frac{\partial v w}{\partial z}= & -\frac{1}{\rho} \frac{\partial p}{\partial y}+\frac{\partial}{\partial x}\left(v \frac{\partial v}{\partial x}+\tau_{x y}\right)+\frac{\partial}{\partial y}\left(v \frac{\partial v}{\partial y}+\tau_{y y}\right) \\
& +\frac{\partial}{\partial z}\left(v \frac{\partial v}{\partial z}+\tau_{z y}\right) \\
\frac{\partial w}{\partial t}+\frac{\partial u w}{\partial x}+\frac{\partial v w}{\partial y}+\frac{\partial w^{2}}{\partial z}=- & \frac{1}{\rho} \frac{\partial p}{\partial z}-g+\frac{\partial}{\partial x}\left(v \frac{\partial w}{\partial x}+\tau_{x z}\right)+\frac{\partial}{\partial y}\left(v \frac{\partial w}{\partial y}+\tau_{y z}\right) \\
& +\frac{\partial}{\partial z}\left(v \frac{\partial w}{\partial z}+\tau_{z z}\right)
\end{aligned}
$$

Where,

$\begin{array}{cll}x, y, z & = & \text { Cartesian coordinates with } z \text { positive vertically upward } \\ g & = & \text { Gravitational acceleration } \\ \tau_{x y}, \text { etc } & = & \text { Sub-grid scale stresses } \\ \rho & = & \text { Density of water } \\ v & = & \text { Kinematic viscosity of water }\end{array}$

\subsubsection{Subgrid and Wall model}

The solution of equation of motion and sub-grid scale effects are very important specially flow near solid surfaces like wall region. Near wall region creates turbulence which gives resistance to flow, the bursting eddies in this region are largely responsible for this large resistance. The standard Smagorinsky eddy viscosity is used for the sub-grid stresses within the flow away from solid boundaries. Wall model is formulated such that it relates near wall flow velocity to wall shear stress on local and instantaneous conditions. The model which is used should reduce the sub-grid eddy viscosity or dynamically determined eddy viscosity coefficient should be used. The Cartesian shear stress components on the wall are shown in terms of velocity in Equation 2,

$$
\begin{array}{rlrl}
\tau_{x y} & =C_{d} \rho V_{1} v_{1}, & & \tau_{x z}=C_{d} \rho V_{1} w_{1} \\
\tau_{y x}=C_{d} \rho V_{1} u_{1}, & & \tau_{y z}=C_{d} \rho V_{1} w_{1} \\
\tau_{z x}=C_{d} \rho V_{1} u_{1}, & & \tau_{y z}=C_{d} \rho V_{1} v_{1}
\end{array}
$$

Where,

$$
\begin{aligned}
& V_{1} \quad=\sqrt{u_{1}^{2}+v_{1}{ }^{2}+w_{1}{ }^{2}} \\
& C_{d} \quad=\quad \text { Model resistance coefficient } \\
& u_{1}, v_{1}, w_{1}=\text { Velocity components at } z=z_{1}
\end{aligned}
$$


The symmetry conditions are required for stresses within flow which is specific for flow near solid wall and Equation 2 does not satisfy it. Thus here mean velocity and mean wall stress equations are used,

$$
C_{d}=\left[\frac{A \ln \left(z_{1} u_{\tau}\right)}{v}+B\right]^{-2}, \frac{k_{s} u_{\tau}}{v} \leq 100 \text { and } \frac{z_{1} u_{\tau}}{v}>10
$$

Where,

$$
\begin{array}{cll}
A, B, C & = & \text { Model coefficient } \\
z_{1} & = & \text { Distance from wall } \\
u_{\tau} & = & \text { Friction velocity } \\
k_{s} & = & \text { Roughness height }
\end{array}
$$

$$
\begin{gathered}
{\left[\frac{z_{1} u_{\tau}}{v}\right]^{-2}, \frac{k_{s} u_{\tau}}{v} \leq 100, \text { and } \frac{z_{1} u_{\tau}}{v} \leq 10} \\
C_{d}=\left[A \ln \frac{z_{1}}{k}+C\right]^{-2}, \quad \frac{k_{s} u_{\tau}}{v}>100
\end{gathered}
$$

where $\mathrm{A}=2.5, \mathrm{~B}=5.2, \mathrm{C}=8.5$.

Equations (2-5) are solved iteratively as a "wall function method" used in two equation turbulence models.

\section{Computation and results}

As an example of application of the method described above, calculation of the flow past a circular cylinder is presented here. In addition to the basic flow past a cylinder in a uniform flow perpendicular to the cylinder axis of infinite extent shown in Fig.1 (a), the flow when the cylinder is placed near a solid surface as shown in Fig.1 (b) such as the case of a pipeline installed near the seabed, has been calculated. The latter is a subject of recent research in offshore engineering and calculations using RANS method has been conducted by [15] which can be compared to the present calculation method.

The region of calculation extends 8 cylinder diameters D upstream, 16D downstream, $8 \mathrm{D}$ above and below the cylinder normal to the flow direction and $2 \mathrm{D}$ in the direction of the cylinder axis for the case of uniform flow. This calculation region is resolved by the rectangular mesh of $127 \times 95 \times 16$ grids with varying spacing made smallest near the cylinder. The minimum spacing is $0.625 \mathrm{D}$ so there are 16 grid points across the diameter of the cylinder. For the case of the cylinder near sea bed, the gap $\mathrm{G}$ between the cylinder and the seabed is $0.35 \mathrm{D}$ as [15] has showed that the effects of the wall become significant. In this case the boundary layer on the seabed develops from the beginning of the calculation region, since a uniform flow is assumed at the upstream end of the calculation region. This corresponds to a case where the pipeline is installed in an area where there is a mound of some height rather than on a flat seabed in open sea, in which case the bottom boundary layer would have developed to the full depth of the sea. The grid spacing in the vertical direction near the bed is taken small but the total number of the grid points is almost the same. The Reynolds number based on D for both calculations is 105 so that the flow is supercritical and the boundary layer on the cylinder becomes turbulent before separating. Fig. 2 shows the calculated flow fields at an instant $t=60 \mathrm{~d} / \mathrm{U} 0$ at which time the flow assumed uniform at initial $t=0$ has fully developed. The figures show only part of the flow near the cylinder so the details can be seen. The symmetric vortex shedding pattern is 
seen in the basic uniform flow case without a wall. In the second case where a solid wall exists, the flow appears as a mixture of the wake behind a cylinder and the flow over an object on a surface. The third case deal with cylinder is resting on bed and flow is passing above the object. There are undulating wake in the area downstream of about 5D but in the region closer to the cylinder, the overall flow is retarded more and the stagnation point on the upstream surface of the cylinder is lower than the center of the cylinder. The flow in the gap is accelerated but for this size of the gap the decrease of the pressure is not significant and overall an upward lift is generated. This average flow effects and the reduced intensity of the vortex shedding appear the main influence of the bed.

(a)
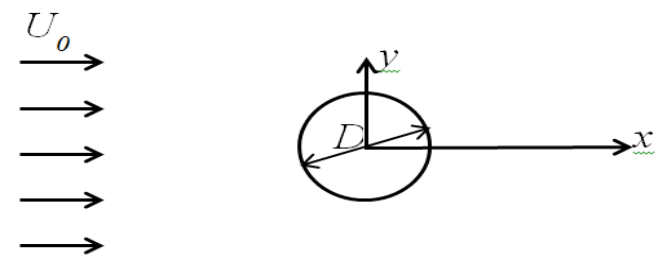

(b)

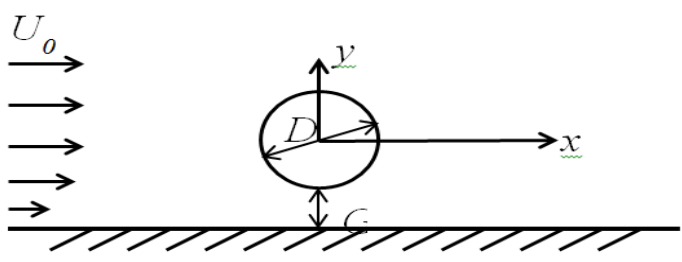

Fig.1. Sample calculation cases, flow past a circular cylinder in uniform flow and near a solid wall.

\subsection{Hydrodynamic forces}

There are a number of hydrodynamic effects that may become important in the design and installation of a pipeline. From the present numerical analysis, we have evaluated the time averaged and fluctuating pressure distributions around the circular cylinder for the three cases of the relative position from the seabed. Fig. 3 shows the distribution of the mean pressure coefficient $C_{p}=\left(P-P_{0}\right) /(\rho / 2) U_{0}^{2}$ as the function of the azimuthal angle $\theta$ defined by the inset in Fig. 3(b). Where $G$ is the gap between the surface of circular cylinder and the plane boundary and $\mathrm{D}$ is the diameter of pipe $\mathrm{P}$ is the mean pressure and $\mathrm{P} 0$ is its value in the undisturbed flow away from the cylinder. The case in the uniform flow without a seabed is the well-studied case and the present results which is done for the Reynolds number typical of the real situation of a pipe line of $1.0 \times 10^{5}$ are compared with the available experimental results at similar Reynolds number and that at lower Reynolds number of 3900 .

The present results follow the experiments on the front surface up to about $\theta=60^{\circ}$ and are between the two experiments. The point where the pressure becomes constant is repeated to the boundary-layer separation. The present results imply the separation a little upstream of the experimental point but the results are generally acceptable. Fig. 3(b) and Fig. 3(c) show the pressure distributions when the cylinder is placed closed to a seabed and right on it. It is seen that the pressure on the upper side of the cylinder decreases and the lower side increases as the pipe is placed near a seabed. On the other hand the pressure on the downstream half of the cylinder surface decreases due to the acceleration through the gap between the cylinder and the seabed. These wall proximity effects imply increased 
upward force and overturning clockwise moment. When the cylinder is placed on the seabed, the gap is completely closed and no acceleration and pressure decrease occur and the up-lifting force acts mostly on the upstream half of the pipe. These dramatic changes in the pressure distribution and the resulting force imply close examination of the flow effect should be made. The fluctuating pressure distributions are shown in Fig. 4 in terms of the root-mean-square $C_{p r m s}$ of the fluctuating pressure coefficient. The fluctuation level is seen to increase as the cylinder is placed near the seabed. The present LES method can be used to examine these important hydrodynamic effects. The above example is only one application of the present simulation method and can be used in various other situations of the flow past objects.

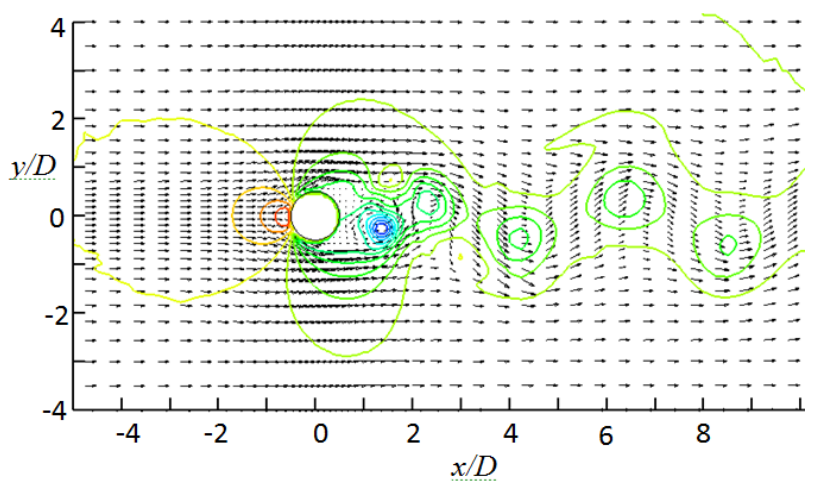

(a) cylinder in uniform flow

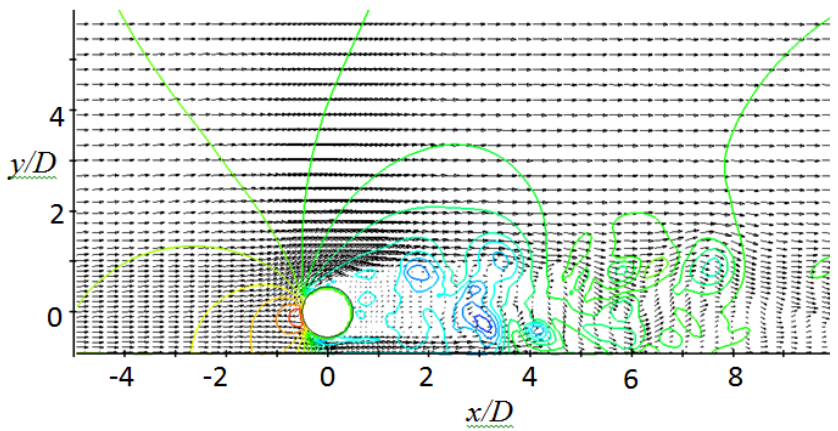

(b) cylinder near solid surface

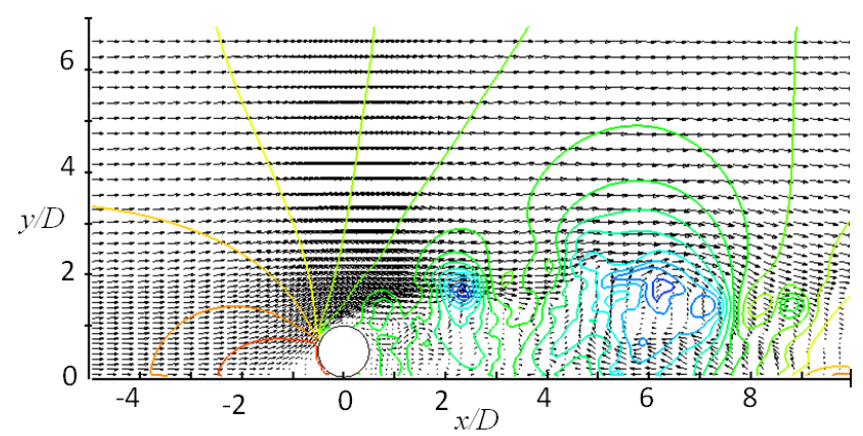

(c) Cylinder at the mud level

Fig. 2. Simulation of offshore pipeline 
(a) in uniform flow

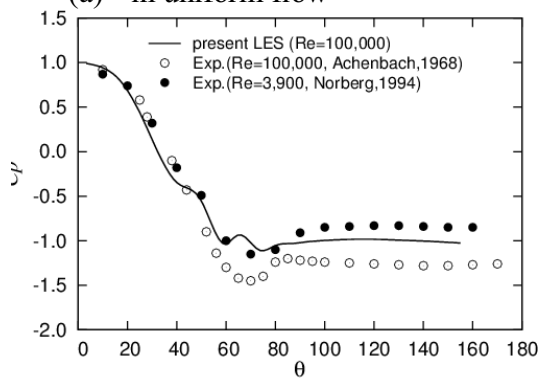

(b) near seabed, $\mathrm{G} / \mathrm{D}=0.35$

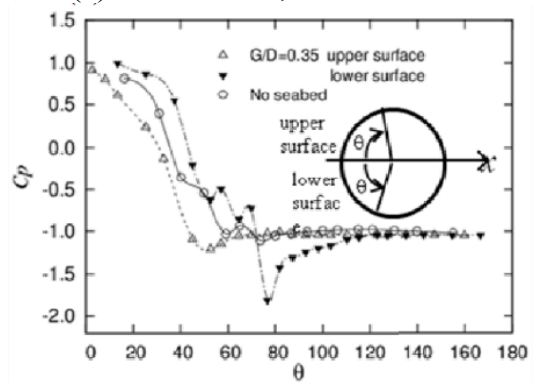

(c) on seabed, $\mathrm{G} / \mathrm{D}=0$

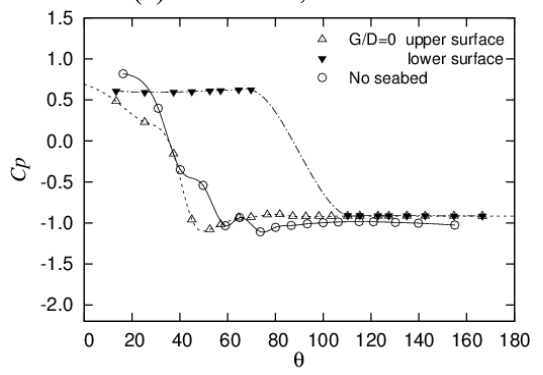

Fig.3. Mean pressure distributions on circular cylinderplaced away from, near and on the seabed

\section{Conclusions}

The results show that the effects of the seabed on the flow characteristics and the loads on the pipe are significant. In the case of without a wall symmetric vortex shedding pattern is seen in the basic uniform flow. The case where a solid wall exists, the flow appears as a mixture of the wake behind a cylinder and the flow over an object on a surface. There are undulating wake in the area downstream of about $5 \mathrm{D}$ but in the region closer to the cylinder, the overall flow is retarded more and the stagnation point on the upstream surface of the cylinder is lower than the center of the cylinder.

The flow in the gap is accelerated but for this size of the gap the decrease of the pressure is not significant and overall an upward lift is generated. This average flow effects and the reduced intensity of the vortex shedding appear the main influence of the bed.The results follow the experimental results up to $\theta=60^{\circ}$ and remain between two experimental results. The study shows separation a little upstream of the experimental point but the results are generally acceptable. When the pipe is placed near a seabed pressure on the upper side of the cylinder decreases and the lower side increases. Pressure decreases on the downstream half of the cylinder due to the acceleration through the gap. The present LES method can be used to examine these important hydrodynamic effects.It was expected that the vortex shedding is weakened since the wall or the seabed makes the wake flow nonsymmetric and the generation of the vortices of alternating directions is naturally reduced. This is what is seen from the present results. More significant result which was not anticipated is that the pressure distribution on the cylinder changes significantly and suddenly as the distance to the wall is reduced. When the gap between the cylinder and the wall is larger than $3.5 \mathrm{D}$, the pressure distribution is not much different from that in the uniform flow without walls, with slight decrease on the upper side and increase on the lower side causing an upward lift force. As the gap is narrowed from 3.5D to zero, the pressure on the front half on the lower side of the cylinder becomes very large, as large as stagnation pressure. It creates large lift and drag forces. This sudden change in the loads is 
very important to consider in the general design of pipelines. It is like a sudden large force an object experiences when it plugs a flow near intake or a drain, and can be very dangerous unless a precaution is taken to avoid a sudden approach to a wall.

\section{References}

[1] M. C. Ong, T. Utnes, L. E. Holmedal, D. Myrhaug, and B. Pettersen, Numerical simulation of flow around a smooth circular cylinder at very high Reynolds numbers. Marine Structures, 22,142-153, (2009)

[2] M. Breuer, Large eddy simulation of the subcritical flow past a circular cylinder: numerical and modeling aspects. Int. J. for Numerical Methods in Fluids, 28,12811302, (1998)

[3] C. Norberg, Experimental investigation of the flow around a circular cylinder: influence of aspect ratio. J. Fluid Mech, 258, 287-316, (1994)

[4] P. Catalano, M.Wang, G. Iaccarino and P. Moin, Numerical simulation of the flow around a circular cylinder at high Reynolds numbers. Int. J. of Heat and Fluid Flow, 24,463-469, (2003)

[5] M.C. Ong, T. Utnes, L.E. Holmedal, , D. Myrhaugand B. Pettersen, , Numerical simulation of flow around a circular cylinder close to a flat seabed at high Reynolds numbers using a $\mathrm{k}-\varepsilon$ model. Coastal Engineering, 57, 931-947, (2010)

[6] J. Johansson, M.P. Nielsen andL.O. Nielsen, Uniform flow around a circular cylinder in the subcritical range - using the self-induced angular moment method turbulence model. The 8th Asia-Pacific Conference on Wind Engineering, Chennai, India, (2013)

[7] F.Tremblay, M. Manhart, and R. Friedrich, LES of Flow around a Circular Cylinder at a Subcritical Reynolds Number with Cartesian Grids. In: FRIEDRICH, R. \& RODI, W. (eds.) Advances in LES of Complex Flows. Springer Netherlands, (2002)

[8] E. Achenbach, Distribution of local pressure and skin friction around a circular cylinder in cross-flow up to $\mathrm{Re}=5 \times 106$. J. Fluid Mech., 34, 625-639, (1968)

[9] S. Li, B.W. Karney and G. Liu, FSI research in pipeline systems - A review of the literature. J. of Fluids and Structures, 57,277-297, (2015)

[10]W. Lin, C. Lin, S. Hsieh and S. Dey, Flow Characteristics around a Circular Cylinder Placed Horizontally above a Plane Boundary. J.of Engineering. Mechanics, 135, 697716, (2009)

[11]A. Nakayama and S.N. Vengadesan, On the influence of numerical schemes and subgrid-stress models on large eddy simulation of turbulent flow past a square cylinder. Int. J. for Numeric. Methods in Fluids, 38, 227-253, (2002)

[12]P. M. Abrahamsen, M. C. Ong, B. Pettersen and D. Myrhaug, Large Eddy Simulations of flow around a smooth circular cylinder in a uniform current in the subcritical flow regime. Ocean Engineering, 77,61-73, (2014)

[13]A. Kulyakhtin, O. Shipilova and M. Muskulus, Numerical simulation of droplet impingement and flow around a cylinder using RANS and LES models. J. of Fluids and Structures, 48, 280-294, (2014)

[14]I. Rodríguez , O. Lehmkuhl, J. Chiva, R. Borrell, and A. Oliva, On the flow past a circular cylinder from critical to super-critical Reynolds numbers: Wake topology and vortex shedding. Int. J. of Heat and Fluid Flow, (2015)

[15]D. Liang and L. Cheng, Numerical modeling of flow and scour below a pipeline in currents: Part I. Flow simulation. Coastal Engineering, 52, 25-42, (2005) 